\title{
Desenvolvimento do olhar e do contato ocular em lactentes de zero a quatro meses de idade
}

\section{The development of looking behavior and eye-contact in breast feeding children aged between 0 and 4 months}

Aline Elise Gerbelli Belini 1

Fernanda Dreux Miranda Fernandes 2
1,2 Laboratório de Investigação Fonoaudiológica em Distúrbios do Espectro Autístico. Departamento de Fisoterapia, Fonoaudiologia e Terapia Ocupacional. Faculdade de Medicina. Universidade de São Paulo. Rua Cipotânea, 51. Cidade Universitária. São Paulo, SP, Brasil. CEP 05.360-000. E-mail: aliluvi@yahoo.com.br

\begin{abstract}
Objectives: to investigate the development of eyecontact and visual behavior in babies aged between 0 and 4 months.

Methods: sixteen normal babies were filmed monthly for 30 minutes, and 11 eleven visual behavior categories observed and transcribed. These were then quantified and treated statistically using the Friedman test $(\alpha=0.050)$ and Wilcoxon's Signed Rank test (Bonferroni's $\alpha=0,010$ ).

Results: there were statistical differences in frequency between the various categories: "looking mother in the eye" $(p<0.001)$, "looking mother in the face" ( $p<0.001)$, "looking at an object" $(p<0.001)$, "looking at the researcher" ( $p<0.001)$, "actively looking at the surroundings" $(p<0.001)$, "passively looking at the surroundings" $(p<0.001)$, "looking at own body" ( $p=0.001)$ and "eyes closed" $(p=0.005)$. Eye-contact was detected in the third week of life in $64.7 \%$ and developed during the first five months of life ( $p=0.010$ in $50 \%$ of the intervals between data collection).

Conclusions: eye-contact can be detected from the neonatal period onwards and develops over the first few months of life. The frequency of the baby's eyecontact with its mother increases longitudinally in the first four months. Other focuses of attention, apart from the mother's eyes, are also fundamental during the early stages of development outside the womb.
\end{abstract}

Key words Nonverbal communication, Infant, newborn, Infant, Child development, Visual perception

\section{Resumo}

Objetivos: investigar o desenvolvimento do contato ocular e do comportamento visual de bebês entre zero e quatro meses.

Métodos: dezessete bebês típicos foram filmados mensalmente por 30 minutos, sendo transcritas 11 categorias de observação do olhar, quantificadas $e$ tratadas estatisticamente através dos testes de Friedman $(\alpha=0,050)$ e dos Postos Sinalizados de Wilcoxon ( $\alpha$ de Bonferroni $=0,010$ ).

Resultados: houve diferenças estatisticamente significantes nas frequências das categorias: "olhar para os olhos da mãe" $(p<0,001)$, "olhar para o rosto da mãe" $(p<0,001)$, "olhar para objeto" $(p<0,001)$, “olhar para a pesquisadora" $(p<0,001)$, "olhar para o ambiente de forma ativa" ( $p<0,001)$, "olhar para o ambiente de forma passiva" ( $p<0,001)$, "olhar para o próprio corpo" $(p=0,001)$ e "olhos fechados" ( $p=0,005)$. Detectou-se o contato ocular na terceira semana de vida $(64,7 \%)$ e comprovou-se sua evolução nos cinco primeiros meses ( $p=0,010 \mathrm{em}$ $50 \%$ dos intervalos entre coletas).

Conclusões: o contato ocular pode ser detectado desde o periodo neonatal e se desenvolve nos primeiros meses de vida. A frequência do contato ocular do bebê com sua mãe aumenta longitudinalmente nos primeiros quatro meses. Existem outros focos de olhar do bebê fundamentais no início do desenvolvimento extrauterino além dos olhos de sua mãe.

Palavras-chave Comunicação não verbal, Recémnascido, Lactente, Desenvolvimento infantil,

Percepção visual 


\section{Introdução}

O conhecimento das capacidades sensoriais em estágios precoces da vida contribuiu para o reconhecimento de que os bebês são seres ativos e com capacidades de adaptação ao meio. A evolução da capacidade visual nos humanos a partir da vida intrauterina tem sido descrita e o conhecimento sobre a cronologia de aquisição de habilidades visuais e seu impacto sobre o desenvolvimento tem sido estabelecido. ${ }^{1-4}$

Comportamentos apresentados por bebês, como o olhar, expressões faciais, vocalizações, monitoramento social, atos comunicativos e prontidão para interação podem ser transcritos em forma de código.5-6

Farroni et al.7e Symons et al. 8 consideram que em situação triádica indivíduos no papel de observadores do olhar têm sensibilidade à direção do olhar da pessoa observada, especialmente quando podem observar os dois olhos. Striano e Stahl9 relatam que, aos três meses, os bebês são sensíveis à interação entre três pessoas mediada pelo contato direto do examinador com eles, olhando mais para a examinadora quando esta coordena afeto e atenção.

Os conhecidos experimentos com a bola vermelha ou outros estímulos indicam a importância de objetos no desenvolvimento sensorial normal do bebê.3,10 Fivaz-Depeursinge et al.11 ressaltam a falta de possibilidades de classificação do olhar do bebê com foco em objetos ou em partes do corpo. Outros autores propõem formas que considerem a interatividade do olhar: "olhar direto", "olhar desviado", "evitação do olhar", "olhar simultâneo interrompido", "olhar simultâneo não interrompido", "olhar adesivo" ou "penetrante".12,13

A literatura indica que ao longo dos primeiros meses de desenvolvimento após o nascimento, o bebê demonstra aprendizado social relativo à visão. ${ }^{14}$ Poucas horas após o nascimento, neonatos aprendem as habilidades de reconhecimento e identificação da face materna. ${ }^{15,16}$ Com sete semanas o contato ocular passa a ter valor social na interação. ${ }^{17}$ Bertin e Striano 18 detectaram aumento do olhar do bebê para o rosto do adulto, quanto mais velho o bebê, entre o primeiro e o terceiro meses de vida.

Ao completar dois meses de vida, o bebê adquire a visão em profundidade com a convergência dos dois olhos, 19,20 passa a dar mais atenção à interação com sua mãe, demonstrando diferenciar seu comportamento social de seu comportamento em relação a objetos $^{2}$ e passa a apresentar o sorriso dirigido ao outro. 20 Ao longo do quarto mês, a literatura enfatiza um salto de percepção do objeto externo pelos bebês. 20,21

De acordo com o conhecimento atual, espera-se que: a) o contato ocular com a mãe esteja presente desde o período neonatal; b) o desenvolvimento do contato ocular ocorra nos primeiros meses de vida, a exemplo de outras áreas cujo desenvolvimento está relacionado ao desenvolvimento do olhar; c) a frequência do contato ocular do bebê com a mãe aumente progressivamente ao longo dos primeiros cinco meses; d) existam outros focos de olhar do bebê, além dos olhos de sua mãe, no início do desenvolvimento extrauterino.

Os objetivos deste trabalho foram: a) avaliar a ocorrência do contato ocular de neonatos com as suas respectivas mães (olhar para os olhos da mãe); b) analisar a evolução do contato ocular do bebê com a mãe nos primeiros meses de vida; c) verificar a frequência de contato ocular do bebê com a mãe ao longo dos primeiros meses de vida; d) verificar a que elementos os bebês dirigem o olhar após o nascimento.

\section{Método}

A pesquisa envolveu 17 bebês que foram seguidos longitudinalmente de zero a quatro meses de vida e que foram selecionados de acordo com os seguintes critérios: a) ausência de malformações ou síndromes congênitas; b) peso ao nascimento maior de $2500 \mathrm{~g}$; c) adequação quanto ao crescimento intrauterino; d) aprovação no rastreamento neonatal para fenilcetonúria; e) aprovação na triagem auditiva neonatal; f) ausência de patologias neonatais que impedissem a alta hospitalar no tempo previsto; g) paridade zero ou um; integração de núcleos familiares completos; h) pais residentes na Grande São Paulo - São Paulo - Brasil. Apenas mãe, pai e filho(s) residiam nos domicílios.

Inicialmente os pais receberam informações gerais sobre a pesquisa. Os prazos definidos para a realização das coletas de dados consideraram uma variação de sete dias a partir do início da segunda quinzena de cada mês de vida dos bebês, sendo: F1 entre o $15^{\circ}$ e o $22^{\circ}$ dias de vida (DV) do bebê; F2 entre o $45^{\circ}$ e $52^{\circ} \mathrm{DV} ; \mathrm{F} 3$ - entre o $75^{\circ}$ e o $82^{\circ} \mathrm{DV} ; \mathrm{F} 4$ - entre o $105^{\circ}$ e o $112^{\circ} \mathrm{DV} ; \mathrm{F} 5$ - entre o $135^{\circ}$ e o $142^{\circ}$ DV. Foram realizadas visitas domiciliares da pesquisadora principal às díades mãe-bebê. $\mathrm{O}$ agendamento das visitas foi efetuado por meio de contatos telefônicos com uma semana de antecedência. A primeira visita tinha duração média de duas horas e as visitas subseqüentes - duração aproximada de uma hora.

As filmagens de cada sujeito foram realizadas 
com câmera de vídeo analógica (modelo Gradiente GCP-155C) e assistidas em ordem sequencial de coleta (F1 a F5). As fitas foram analisadas por intervalos sequenciais de 30 segundos de duração - 60 intervalos por filmagem (originando 5100 intervalos de 30 segundos analisados, considerando-se as 85 filmagens, cinco de cada um dos 17 sujeitos).

Antes do início de cada filmagem, a pesquisadora solicitava à mãe que agisse da forma mais natural e usual possível e que realizasse a (s) atividade(s) cotidianamente prevista(s) com o bebê para aquele momento. As gravações tiveram duração de cerca de 30 minutos e a aproximação e o ângulo de filmagem foram dinamicamente ajustados de forma a enquadrar os rostos do bebê e da mãe. Durante as filmagens, buscou-se manter o enquadramento e a aproximação mínima que propiciasse a nitidez necessária à observação dos olhos do bebê.

Cada um dos intervalos foi integral e continuamente assistido, sendo registradas as "categorias de observação do olhar" (COO), ocorridas quando os bebês estavam em estado de alerta (determinado de acordo com os critérios de Brazelton 2 ). As onze COO dizem respeito aos alvos do olhar dos bebês e foram determinadas para essa pesquisa (Tabela 1).

Para o registro das categorias de observação do olhar (COOs), levou-se em conta sua ocorrência em cada intervalo de 30 segundos. O material transcrito nos protocolos de registro apropriados foi organizado pelo Excel e analisado por software especialmente formulado (Sistema Consolidador de Informações Coletadas), que contabilizou a frequência de manifestação de cada categoria de observação do olhar, ou seja, o percentual de intervalos em que ocorreu cada uma das COOs em relação ao total de intervalos em que o bebê esteve em estado de alerta.

Vinte por cento das filmagens foram submetidas à análise por duas fonoaudiólogas com larga experiência na observação de bebês. As filmagens a serem julgadas e a sequência de apresentação dos sujeitos foram sorteadas. Ocorreram sete encontros, sendo o primeiro deles uma sessão de treinamento. Estiveram presentes as juízas e a primeira autora, encarregada da cronometragem e determinação dos intervalos. As juízas assistiram a cada intervalo completo e fizeram seus registros em protocolos individuais. As três primeiras filmagens sorteadas

\section{Tabela 1}

Categorias de observação do olhar (COOs) e síntese das respectivas definições.

\begin{tabular}{|c|c|c|}
\hline Sigla & Nome & Síntese da definição \\
\hline OMO & Olhar para os olhos da mãe & $\begin{array}{l}\text { Dirigir o olhar aos olhos ou a um dos olhos da mãe. Olho a } \\
\text { olho mútuo, contato ocular propriamente dito. }\end{array}$ \\
\hline OMR & Olhar para o rosto da mãe & $\begin{array}{l}\text { Dirigir o olhar ao rosto ou cabelos da mãe. } \\
\text { O comportamento da mãe auxilia na distinção com o OMO. }\end{array}$ \\
\hline OMC & Olhar para o corpo da mãe & $\begin{array}{l}\text { Fixar ou passar o olhar pelo corpo da mãe: membros, ombros, } \\
\text { tronco, mama, mãos ou quando esta está de costas, de lado, } \\
\text { ou movimenta-se pelo ambiente, distante do bebê. }\end{array}$ \\
\hline OAA & Olhar para o ambiente de forma ativa & Olhar vivo, dirigido à cena ambiental. \\
\hline OAP & Olhar para o ambiente de forma passiva & $\begin{array}{l}\text { Olhar apagado; "perdido" pelo ambiente, não focaliza } \\
\text { elementos específicos da cena, nem a cena em si. }\end{array}$ \\
\hline OJ & Olhar para objeto(s) & $\begin{array}{l}\text { Ativo quando dirigido a objetos pequenos. Pode ser passivo } \\
\text { quando dirigido a objetos grandes, Olhar para peças de } \\
\text { vestuário próprias ou da mãe, mesmo que vestidas, quando } \\
\text { atento às propriedades físicas delas. }\end{array}$ \\
\hline OPC & Olhar para o próprio corpo & $\begin{array}{l}\text { Fixar ou passar o olhar pelo próprio corpo (membros ou } \\
\text { tronco). }\end{array}$ \\
\hline & & Olhar para a própria imagem no espelho. \\
\hline OP & Olhar para a pesquisadora & $\begin{array}{l}\text { Fixar ou passar o olhar pela pesquisadora (corpo ou rosto). } \\
\text { Dirigir o olhar à câmera filmadora ou a seus componentes. }\end{array}$ \\
\hline O० & Olhar para outra pessoa & Dirigir o olhar a quarta pessoa da cena. \\
\hline OF & Olhos fechados & $\begin{array}{l}\text { Reação a estímulos externos, internos, interação materna. } \\
\text { Manter os olhos fechados, incluindo piscada prolongada. }\end{array}$ \\
\hline AFO & Abrir e fechar os olhos & $\begin{array}{l}\text { Manifestação do estado de sonolência. Piscar os olhos } \\
\text { repetidas vezes, como reação a estímulos externos (visuais, } \\
\text { auditivos ou sinestésicos) ou visando defender-se deles. }\end{array}$ \\
\hline
\end{tabular}


foram utilizadas para compatibilização de análises. A concordância entre os observadores foi testada utilizando o Teste de Concordância de Kendall. As comparações entre as analisadoras indicou fraca concordância para as categorias 'olhar para o rosto da mãe' (OMR) $(0,012 ; p=0,811)$; 'olhar para o corpo da mãe' (OMC) $(0,005 ; p=0,923)$; 'olhar para os olhos da mãe' (OMO) $(0,031 ; p=0,592)$; 'olhar para objetos' (OJ) $(0,020 ; p=0,711) \mathrm{e}$ 'olhar para a pesquisadora' (OP) $(0,015 ; p=0,779)$; considerável para as categorias 'abrir e fechar os olhos' (AFO) $(0,363 ; p=0,002)$ e 'olhar o próprio corpo' (OPC) $(0,209 ; p=0,028)$; moderada para 'olhar para o ambiente de forma ativa' (OAA) $(0,489 ; p<0,001)$, e substancial para 'olhar para o ambiente de forma passiva' (OAP) $(0,618 ; p<0,001)$ e 'olhos fechados' (OF) $(0,775 ; p<0,001)$.

As diferenças entre os valores médios das COOs nos diferentes momentos de avaliação foram testadas aplicando-se o Teste de Friedman, sendo adotado um nível de significância de 5\%. Os valores médios dos COOs nos diferentes momentos foram comparados par a par em cada momento, aplicando-se o Teste dos Postos Sinalizados de Wilcoxon, com a Correção de Bonferroni $(\alpha=0,010)$.

A pesquisa foi aprovada pela Comissão de Ética para Pesquisa (CAPPesq Hospital das Clínicas da Faculdade de Medicina da Universidade de São Paulo - HCFMUSP / parecer número 445/04). Os pais dos sujeitos assinaram o Termo de Consentimento Livre e Esclarecido, aprovado pela mesma comissão.

\section{Resultados}

Entre os bebês estudados, 10 (58,8\%) eram do sexo masculino e $7(41,2 \%)$ do sexo feminino. Todas as mães e as crianças tiveram acesso ao atendimento pré-natal e à puericultura, respectivamente. As mães dos bebês tinham idade média de 30,8 (variação: 20 47) anos e os pais, 37 (variação: 28-46) anos. A maioria das mães $(64,7 \%)$ e dos pais $(70,6 \%)$ havia concluído o ensino superior.

No período neonatal (F1), 100\% dos sujeitos apresentaram alguma ocorrência de olhos fechados (OF) e de olhar para o ambiente de forma passiva (OAP); 94,1\% dos sujeitos apresentaram as COOs OAA, OMR e OMC; $64,7 \%$ da amostra apresentaram o olhar dirigido à pesquisadora $(\mathrm{OP})$ e aos olhos da mãe (OMO); 58,8\% da amostra apresentaram AFO; 47,1\% da amostra apresentaram OPC; $23,53 \%$ dos sujeitos apresentaram olhar dirigido a objetos (OJ) e 5,9\% da amostra apresentaram olhar para outra pessoa (OO).
No segundo mês de vida (F2), as COOs OP, OAP, OMR e OMC foram detectadas nas filmagens de $100 \%$ dos sujeitos. As COOs OF e OAA foram manifestas por $94,1 \%$ dos sujeitos; o olhar para os olhos da mãe (OMO) ocorreu em 88,24\% dos sujeitos; o olhar dirigido a objetos (OJ) observou-se em $76,5 \%$ da amostra; AFO ocorreu nas filmagens de $47,1 \%$ dos sujeitos; OPC em $41,2 \%$ da amostra e OO em $17,6 \%$.

No terceiro momento (F3), constatou-se que $100 \%$ dos bebês com dois meses e meio de idade olharam para a pesquisadora (OP), para o ambiente de forma ativa (OAA) e passiva (OAP), para o rosto (OMR) e para o corpo da mãe (OMC); 94,1\% deles apresentaram ocorrências das COOs OF, OJ e OMO; $70,6 \%$ deles OPC; $35,3 \%$ AFO e $11,8 \%$ apresentaram OO.

No momento F4, 100\% dos bebês, aos três meses completos, apresentaram as COOs OF, OJ, OP, OAA, OAP, OMR, OMO e OMC; 70,6\% olharam para o próprio corpo (OPC) durante a filmagem; $52,9 \%$ abriram e fecharam os olhos (AFO) e $11,8 \%$ olharam para outra pessoa (OO).

No momento final (F5), aos quatro meses de vida, $100 \%$ dos bebês apresentaram as COOs OF, OJ, OP, OAA, OAP, OMR, OMO e OMC; $94,1 \%$ manifestaram o olhar para o próprio corpo (OPC); $58,8 \%$ abriram e fecharam os olhos (AFO) e $17,6 \%$ olharam para outra pessoa (OO). No conjunto dos cinco momentos pesquisados observou-se a seguinte frequência de COOs: OAA (48,2\%), OMR (43,5\%), OMC $(34,7 \%)$, OAP $(33,2 \%)$, OP $(31,5 \%)$, OJ $(20,6 \%)$, OF $(19,0 \%)$, OMO $(18,2 \%)$, OPC $(5,3 \%)$, AFO $(3,3 \%)$, OO $(0,8 \%)$ como se observa na Tabela 2.

Os valores de significância encontrados pelo Teste de Friedman na comparação concomitante dos cinco momentos para cada uma das COOs evidenciaram motivação para a comparação par a par entre os momentos em oito das categorias: OAA, OMR, OAP, OP, OJ, OF, OMO e OPC (Tabela 2).

A comparação entre os cinco momentos de coleta de dados, através do Teste dos Postos Sinalizados de Wilcoxon, com a aplicação da Correção de Bonferroni, evidenciou em quais intervalos de coleta a amostra apresentou modificações estatisticamente significantes nas diferentes COOs ao longo de seu desenvolvimento (Tabela 3 ).

As quatro COOs mais fundamentais para a determinação do desenvolvimento longitudinal do olhar, nos primeiros meses de bebês em desenvolvimento típico, são: OP e OJ, já que nelas se observa o maior número de intervalos entre coletas com $p$-valor estatisticamente significante. 
Os intervalos em que o desenvolvimento do olhar foi mais evidente, ou seja, os intervalos nos quais houve maior número de COOs com diferenças estatisticamente significantes de frequência $(75 \%$ das COOs) foram: F1 x F4 e F1 x F5.

Tabela 2

Frequência de manifestação das categorias de observação do olhar (COOs) na amostra.

\begin{tabular}{|c|c|c|c|c|c|c|c|}
\hline \multirow[t]{2}{*}{$\mathrm{COO}$} & \multicolumn{5}{|c|}{ Momento } & \multirow[t]{2}{*}{ Valor Médio } & \multirow[t]{2}{*}{$p * *$} \\
\hline & $\mathrm{F} 1$ & $\mathrm{~F} 2$ & F3 & $\mathrm{F} 4$ & F5 & & \\
\hline OAA & 27,82 & 39,76 & 48,94 & 60,00 & 64,24 & 48,15 & $<0,001$ * \\
\hline OMR & 20,94 & 45,24 & 53,12 & 53,00 & 45,12 & 43,48 & $<0,001$ * \\
\hline OMC & 46,35 & 30,47 & 30,06 & 35,24 & 31,59 & 34,74 & 0,161 \\
\hline OAP & 47,71 & 42,18 & 31,47 & 18,41 & 26,06 & 33,17 & $<0,001$ * \\
\hline OP & 4,59 & 23,29 & 31,24 & 41,53 & 56,59 & 31,45 & $<0,001$ * \\
\hline OJ & 1,82 & 4,71 & 14,18 & 35,71 & 46,59 & 20,60 & $<0,001$ * \\
\hline OF & 25,59 & 13,00 & 17,71 & 16,82 & 21,88 & 19,00 & $0,005^{*}$ \\
\hline OMO & 2,88 & 16,41 & 25,94 & 26,35 & 19,59 & 18,23 & $<0,001 *$ \\
\hline OPC & 4,47 & 2,06 & 3,47 & 7,41 & 9,12 & 5,31 & $<0,001$ * \\
\hline AFO & 5,24 & 2,71 & 1,71 & 2,41 & 4,18 & 3,25 & 0,106 \\
\hline OO & 0,65 & 1,53 & 0,88 & 0,35 & 0,65 & 0,81 & 0,809 \\
\hline
\end{tabular}

OAA=olhar para o ambiente de forma ativa; OMR=olhar para o rosto da mãe; OMC=olhar para o corpo da mãe; $\mathrm{OAP}=$ olhar para o ambiente de forma passiva; $\mathrm{OP}=\mathrm{olh}$ ar para a pesquisadora; OJ=olhar para objeto(s); OF=olhos fechados; $\mathrm{OMO}=$ olhar para os olhos da mãe; OPC=olhar para o próprio corpo; $\mathrm{AFO=abrir}$ e fechar os olhos; OO=olhar para outra pessoa; F1=primeira filmagem; F2=segunda filmagem; F3=terceira filmagem; F4=quarta filmagem; $\mathrm{F} 5=$ quinta filmagem; *Valor estatisticamente significante $(p<0,05) ; * *$ Teste de Friedman.

Tabela 3

Valores de $p$ relativos ao Teste dos Postos Sinalizados de Wilcoxon empregado na comparação entre os diferentes intervalos de coleta de dados para cada categoria de observação do olhar (COO); indicados os valores de $p$ estatisticamente significantes - inferiores ao valor alfa de Bonferroni $(0,010)$.

\begin{tabular}{|c|c|c|c|c|c|c|c|c|}
\hline $\begin{array}{l}\text { Intervalos } \\
\text { de coleta }\end{array}$ & OAA & OMR & OAP & OP & OJ & OF & OMO & OPC \\
\hline $\mathrm{F} 1 \times \mathrm{F} 2$ & 0,011 & $0,002 *$ & 0,309 & $<0,001 *$ & 0,050 & 0,004 * & 0,001 * & 0,154 \\
\hline $\mathrm{F} 1 \times \mathrm{F} 3$ & $0,002 *$ & $<0,001$ * & 0,044 & $<0,001 *$ & $<0,001 *$ & 0,113 & $<0,001 *$ & 0,975 \\
\hline $\mathrm{F} 1 \times \mathrm{F} 4$ & $<0,001 *$ & $<0,001$ * & $0,002 *$ & $<0,001 *$ & $<0,001 *$ & 0,015 & $<0,001 *$ & 0,209 \\
\hline $\mathrm{F} 1 \times \mathrm{F} 5$ & $<0,001$ * & 0,002 * & 0,004 * & $<0,001$ * & $<0,001$ * & 0,244 & $<0,001 *$ & 0,055 \\
\hline $\mathrm{F} 2 \times \mathrm{F} 3$ & 0,031 & 0,214 & 0,015 & 0,008 * & 0,004 * & 0,052 & 0,070 & 0,167 \\
\hline $\mathrm{F} 2 \times \mathrm{F} 4$ & 0,003 * & 0,205 & $<0,001^{*}$ & 0,006 * & $<0,001 *$ & 0,356 & 0,006 * & 0,024 \\
\hline $\mathrm{F} 2 \times \mathrm{F} 5$ & 0,001 * & 0,943 & 0,010 * & $<0,001$ * & $<0,001 *$ & 0,018 & 0,244 & 0,003 * \\
\hline $\mathrm{F} 3 \times \mathrm{F} 4$ & 0,017 & 0,943 & 0,011 & 0,026 & 0,002 * & 0,653 & 0,924 & 0,010 * \\
\hline $\mathrm{F} 3 \times \mathrm{F} 5$ & $0,002 *$ & 0,136 & 0,256 & $<0,001 *$ & $<0,001 *$ & 0,352 & 0,124 & $<0,001$ * \\
\hline $\mathrm{F} 4 \times \mathrm{F} 5$ & 0,196 & 0,093 & 0,093 & $0,005^{*}$ & 0,037 & 0,246 & 0,061 & 0,113 \\
\hline
\end{tabular}

OAA=olhar para o ambiente de forma ativa; OMR=olhar para o rosto da mãe; OAP=olhar para o ambiente de forma passiva; OP=olhar para a pesquisadora; OJ=olhar para objeto(s); OF=olhos fechados; OMO=olhar para os olhos da mãe; $\mathrm{OPC}=$ olhar para o próprio corpo; F1=primeira filmagem; F2=segunda filmagem; F3=terceira filmagem; F4=quarta filmagem; F5=quinta filmagem; *Valor estatisticamente significante $(p=0,010)$. 
As Figuras 1 e 2 mostram a distribuição de freqüência da presença das categorias de olhar OAA, OP, OJ, OPC e OMR, OAP, OF, OMO, respectivamente, nos cinco diferentes momentos de avaliação. As curvas de ocorrência das oito categorias de olhar apresentaram duas características predominantes: a) o aumento ao longo dos meses - o que pode ser observado na Figura 1 e b) a oscilação ao longo dos meses - o que pode ser observado na Figura 2.

\section{Figura 1}

Ocorrências médias das categorias de observação do olhar (COO) longitudinalmente ascendentes.

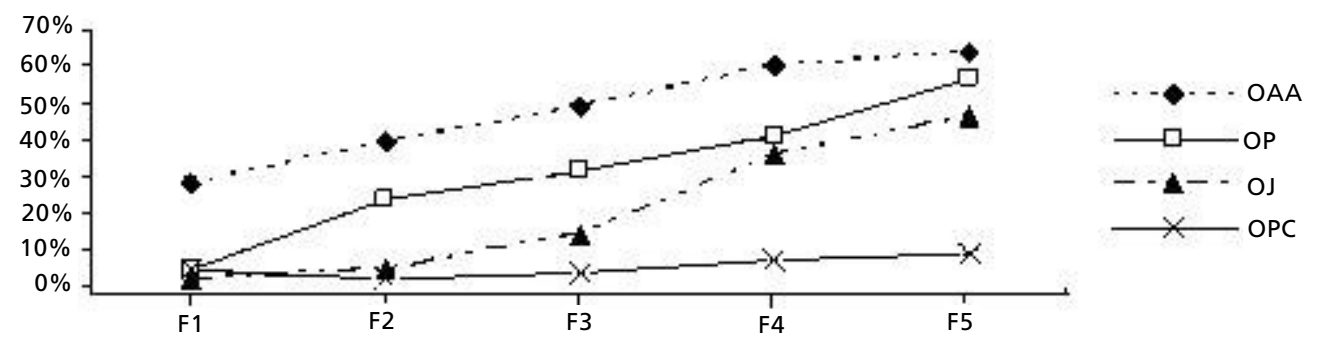

$\mathrm{OAA}=\mathrm{olhar}$ para $\mathrm{o}$ ambiente de forma ativa; $\mathrm{OP=Olhar}$ para a pesquisadora; $\mathrm{OJ}=\mathrm{olhar}$ para objeto(s); OPC=olhar para $\mathrm{O}$ próprio corpo; $F 1=$ primeira filmagem; F2=segunda filmagem; $F 3=$ terceira filmagem; F4=quarta filmagem; F5=quinta filmagem.

Figura 2

Ocorrências médias das categorias de observação do olhar (COO) com oscilação longitudinal.

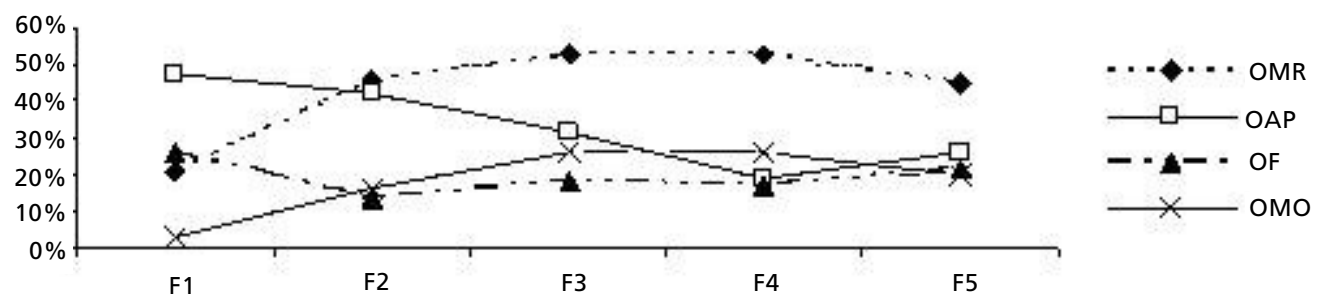

OMR=olhar para o rosto da mãe; $\mathrm{OAP}=\mathrm{olhar}$ para o ambiente de forma passiva; $\mathrm{OF}=\mathrm{olhos}$ fechados; $\mathrm{OMO}=\mathrm{olhar}$ para os olhos da mãe; F1=primeira filmagem, F2=segunda filmagem; F3=terceira filmagem; F4=quarta filmagem; F5=quinta filmagem.

\section{Discussão}

Este estudo teve como objetivo principal investigar o desenvolvimento do contato ocular de bebês saudáveis, dos zero aos quatro meses, em situações cotidianas com suas mães. Foi detectada existência do contato ocular dos neonatos com as mães, já às duas semanas de vida, e foi evidenciada a evolução desse contato nos primeiros meses do desenvolvi- mento. $\mathrm{O}$ aumento da frequência do contato ocular do bebê com sua mãe ao longo dos primeiros cinco meses de vida foi parcialmente confirmado, observando-se aumento estatisticamente significante do contato ocular do primeiro para o segundo mês e, a partir de então, sendo necessário intervalo de dois meses para encontrarem-se níveis estatisticamente significantes de aumento, até o quarto mês. Entre o quarto e o quinto meses, detectou-se estabilidade 
estatística da freqüência do contato ocular, apesar da diminuição da frequência do 'olhar para os olhos da mãe'.

No conjunto dos primeiros cinco meses de seu desenvolvimento, os sujeitos, em alerta, dirigiram seu olhar especialmente ao 'ambiente de forma ativa' (OAA) e ao 'rosto da mãe' (OMR). Outros focos frequentes do olhar dos sujeitos foram o 'corpo da mãe' (OMC), o 'ambiente de forma passiva' (OAP) e a 'pesquisadora' (OP). Os 'objetos' (OJ) foram o foco do olhar imediatamente mais frequente do que os 'olhos da mãe' (OMO). Longitudinalmente, em todos os intervalos pesquisados, a amostra apresentou aumento da frequência do olhar dirigido ao "ambiente" de forma ativa (OAA), à 'pesquisadora' (OP) e aos 'objetos' $(\mathrm{OJ})$.

Os resultados do presente estudo estão de acordo com as evidências científicas de que o contato ocular pode estar presente desde o período neonatal. A observação da categoria 'olhar para os olhos da mãe' (OMO) foi manifestada em um elevado percentual de bebês já na primeira filmagem, ou seja, durante a terceira semana de vida. Esses resultados mostram a presença da habilidade de reconhecer a face materna já nos primeiros dias de vida. 15

As diferenças estatisticamente significantes encontradas entre as frequências de manifestação de 'olhar para os olhos da mãe' (OMO) entre o primeiro mês de vida e todos os demais pesquisados e entre o segundo e o quarto meses parecem relacionar-se ao fato de que a evolução das possibilidades motoras do bebê permite progressivamente o ajuste visual e a expansão do olhar para o mundo. $2-4$

Houve significância estatística na comparação concomitante das frequências da categoria 'olhar para os olhos da mãe' (OMO) ao longo do período de acompanhamento, indicando o desenvolvimento do contato ocular nos primeiros meses de vida dos bebês estudados. Esse achado parece fundamental em vista dos trabalhos referentes ao tema do contato ocular, que enfatizam sua importância e manifestação já ao início da vida, porém raramente oferecem parâmetros de desenvolvimento ou de modificações longitudinais, restringindo-se a algumas publicações que apresentam diferenças entre grupos de bebês com diferentes faixas etárias. ${ }^{18,22}$ Das onze categorias de observação do olhar, oito $(72,7 \%)$ tiveram diferenças estatisticamente significantes entre suas frequências, na comparação global do período, o que indica que há desenvolvimento do contato ocular e do comportamento visual nos primeiros cinco meses do desenvolvimento dos sujeitos.

Confirmando a hipótese de que a frequência do contato ocular do bebê com sua mãe aumenta longitudinalmente nos primeiros cinco meses, houve um aumento significativo da frequência do 'olhar para os olhos da mãe' (OMO) entre o primeiro mês e os demais momentos pesquisados. Detectou-se em valores absolutos, aumento das frequências de OMO na amostra até o quarto mês, porém, a partir do segundo mês foi necessário intervalo de dois meses para que esse aumento fosse significante estatisticamente (de F2 para F4).

A diminuição da frequência da categoria de olhar 'olhar para os olhos da mãe' (OMO) entre o quarto e o quinto meses representa estabilidade do ponto de vista estatístico. ${ }^{18}$ É possível que tal evolução ocorra acompanhando a evolução visual do bebê1,19 e também o desenvolvimento de seu contato. $12 \mathrm{O}$ valor social do contato ocular dos bebês considerando a reação das mães pareceu ocorrer desde mais cedo do que o esperado, de acordo com a literatura, 17 sendo registrado, em muitas duplas, a partir da terceira semana de vida.

Por fim, os dados confirmam a hipótese da existência de outras categorias de observação do olhar fundamentais no início do desenvolvimento humano, além do 'olhar para os olhos da mãe', foi confirmada. Alguns autores trabalham apenas com 'olhar para a mãe' sem diferenciá-lo em 'rosto', 'olho', 'corpo', ou consideram o contato ocular como olhar para a face da mãe.5,6 As diferenças estatisticamente significantes encontradas quanto à frequência de manifestação das categorias OMO, OMR e OMC na amostra, na comparação concomitante entre os cinco momentos pesquisados, realça a importância da distinção entre elas. As médias gerais de ocorrência das categorias de observação do olhar indicaram que a categoria 'olhar para os olhos da mãe' aparece apenas em oitavo lugar, sendo mais frequente que 'olhar para o próprio corpo' (OPC), 'abrir e fechar os olhos' (AFO), duas das categorias com efeito solo.

Em todos os intervalos entre as filmagens, exceto entre os momentos F1 e F2 e entre F4 e F5, houve um significante aumento da frequência da ocorrência da categoria 'olhar para objeto(s)' (OJ), sugerindo que os objetos começaram a tornar-se o foco do olhar dos bebês a partir do terceiro mês, período anterior ao descrito na literatura. 20,21

$\mathrm{O}$ 'olhar para a pesquisadora' (OP) foi a única categoria cuja frequência apresentou aumento estatisticamente significante em 90\% dos intervalos consecutivos de coleta. Esses resultados sugerem que a categoria 'olhar para a pesquisadora' (OP) possa constituir um importante indicador para o estudo do desenvolvimento visual nos primeiros 
meses de vida. O 'olhar para a pesquisadora' (OP) parece também ter significado quanto aos aspectos social e afetivo do desenvolvimento. O paralelismo das curvas de frequência do 'olhar para a pesquisadora' (OP) e do 'olhar para o ambiente de forma ativa' (OAA) ao longo de todo o período pode indicar a importância que o pesquisador (o 'outro') tem para o bebê no ambiente para o qual este ativamente olha.

Situações de oferta triádica $7-9,11$ acabaram por ocorrer devido à presença da pesquisadora, que, neste estudo, teve não apenas a função de analisadora, mas compôs a cena da coleta, inevitavelmente introduzindo a si e ao equipamento de filmagem nas observações realizadas. A apresentação da face da pesquisadora aos bebês durante a filmagem não foi controlada, ocorrendo de forma casual e, provavelmente respondendo ao olhar direto lançado pelo bebê, assim como motivando sua repetição após a primeira ocorrência, até a habituação visual do bebê, ou seu interesse por outros focos. 16

As categorias 'olhos fechados' (OF) e 'abrir e fechar os olhos' (AFO) indicam o "não olhar", contraponto do "olhar concretizado", objetivo deste estudo. Pareceu importante detectar como os bebês se comportam quanto ao fechamento ocular em diversos momentos do desenvolvimento inicial. 'Olhos fechados' (OF) poderia ser uma categoria sugestiva de interação diminuída, o que não se confirmou, por exemplo, nas situações em que o bebê fechou os olhos ao prever a repetição de ação lúdica da mãe. A diminuição da frequência da categoria 'olhos fechados' (OF) entre o primeiro e o segundo meses, estatisticamente significante, por outro lado, pode indicar concordância com os autores, supondo-se que os bebês tornem-se mais interativos no segundo mês em comparação com o primeiro. 6,17

A partir do método utililizado, foram considerados fundamentais: a avaliação de bebês por meio da observação comportamental sistematizada, o registro em vídeo das trocas comunicativas, permitindo a microanálise dos processos, a consideração dos aspectos emocionais do desenvolvimento 6 e o uso de estímulos dinâmicos e naturais em detrimento dos controlados. 10

\section{Referências}

1. Hamilton R, Dudgeon J, Bradnam MS, MacTier H. Development of the electoretinogram between 30 and 50 weeks after conception. Early Human Dev. 2005; 81: 4614.
O instrumento apresentado e aplicado nesse estudo poderá constituir uma opção para a avaliação do desenvolvimento comunicativo do neonato e do lactente, ou ser adaptado para compor instrumentos que visem a investigação precoce do laço mãe-bebê.

A interpretação dos dados fornecidos por este instrumento poderá ser melhor aproveitada se enriquecida pelas observações qualitativas das quais são derivados os dados quantificáveis. A observação atenta de cada bebê e de cada díade pode, mais do que indicar o enquadramento ou não na normalidade, permitir a detecção de aspectos da evolução daquele bebê em seu contexto.

A comunicação não verbal pode ser acessada através de instrumentos como este, baseados na observação atenta do lactente e de sua mãe em situações naturalísticas. A interação materno-filial fornece muitos dados valiosos para o estudo do desenvolvimento humano, além dos tratados nesse trabalho. Novas investigações junto a díades mãebebê de diferentes culturas ou outros níveis socioeconômicos podem indicar similaridades e diferenças que favoreçam a abrangência diagnóstica de instrumentos como o aqui proposto. Ampliações desse estudo com maior número de bebês em desenvolvimento típico, ou investigações junto a populações de risco para o desenvolvimento ou com alterações já detectadas, podem contribuir para a prevenção, para a determinação dos focos de intervenção, e auxiliar no acompanhamento do bebê e da interação da dupla.

Este trabalho fornece informações pioneiras numa área de grande interesse na atualidade, na qual ainda há muito que se pesquisar. Os resultados aqui obtidos, porém, compõem um primeiro estudo, cujas dimensões não permitem a formulação de parâmetros e que deve ser olhado com cautela quanto à generalização dos achados.

\section{Agradecimentos}

Esta pesquisa recebeu apoio financeiro da Fundação de Amparo à Pesquisa do Estado de São Paulo Fapesp, através de Bolsa de Mestrado (04/03998-3). Agradecemos a Cibelle Albuquerque de la Higuera Amato e Silvia Maria Oller do Nascimento Marchi pela colaboração como juízas neste estudo.

2. Brazelton TB. O bebê: parceiro na interação. In: Brazelton TB, Cramer B, Kreisler L, Schappi R, Soulé M. A dinâmica do bebê. São Paulo: Artes Médicas; 1987. p. 9-23. 
3. Klaus M, Klaus P. Expressões faciais. In: Klaus M, Klaus P. O surpreendente recém-nascido. Porto Alegre: Artes Médicas; 1989. p. 83-96

4. Alexander R, Boheme R, Cupps B. Normal Development of Functional Motor Skills: the first year of life. Texas: Therapy Skill Builders; 1993.

5. Laucht M, Esser G, Schmidt MH. Differential development of infants at risk for psychopathology: the moderating role of early maternal responsivity. Dev Med Child Neurol. 2001; 43: 292-300

6. Legerstee M, Varghese $J$. The role of maternal affect mirroring on social expectancies in three-month-old infants. Child Dev. 2001; 72: 1301-13.

7. Farroni T, Csibra G, Simion F, Johnson MH. Eye contact detection in humans from birth. PNAS. 2002; 99: 9602-5.

8. Symons LA, Lee K, Cedrone CC, Nishimura M. What are you looking at? Acuity for triadic eye gaze. J Gen Psychol. 2004; 131: 451-69.

9. Striano T, Stahl D. Sensitivity to triadic attention in early infancy. Develop Scie. 2005; 8: 333-43.

10. Dibiase J, Einspieler C. Can spontaneous movements be modulated by visual and acoustic stimulation in threemonth old infants? J Earl Hum Dev. 2002; 68: 27-37.

11. Fivaz-Depeursinge E, Favez N, Lavanchy C, De Noni S, Frascarolo F. Four-month-olds make triangular bids to father and mother during trilogue play with still-face. Social Dev. 2005; 14: 361-78.

12. Crown CL, Feldstein S, Jasnow MD, Beebe B, Jaffe J. The cross-modal coordination of interpersonal timing: sixweek-olds infants' gaze with adults vocal behavior. J Psycholing Res. 2001; 31: 1-23.

Recebido em 5 de janeiro de 2009

Versão final apresentada em 21 de outubro de 2009

Aprovado em 19 de novembro de 2009
13. Golse B. Autisme Infantile: despiste et prévention. Psychiatr Enfant. 2003; XLVI: 2.

14. Vaish A, Striano T. Is visual reference necessary? Contributions of facial versus vocal cues in 12-month-olds' social referencing behavior. Develop Scie. 2004; 7: 261-9.

15. Walton GE, Armstrong ES, Bower TGR. Faces as forms in the world of the newborn. Infant Behav Develop. 1997; 20 : 537-43.

16. Farroni T, Massaccesi S, Menon E, Johnson MH. Direct gaze modulates face recognition in young infants. Cogntinion. 2007; 102: 396-404.

17. Haith MM, Bergman T, Moore M. Eye contact and face scanning in early infancy. Science. 1977; 198: 853-5.

18. Bertin E, Striano T. The still-face response in newborn, 1.5and 3-month-old infants. Infant Behav Develop. 2006; 29 : 294-7.

19. Lavelli M, Fogel A. Developmental changes in motherinfant face-to-face communication: birth to three months. Develop Psychol. 2002; 3: 288-305.

20. Lavelli M, Fogel A. Developmental changes in the relationship between the infant's attention and emotion during early face-to-face communication: the 2-month transition. Develop Psychol. 2005; 41: 265-80.

21. Carey S, Williams T. The role of object recognition in young infants' object segregation. J Exp Child Psychol. 2001; 78: 55-60.

22. Blass EM, Camp CA. The ontogeny of face recognition: eye contact and sweet taste induce face preference in 9- and 12-week old human infants. Develop Psychol. 2001; 37 : 762-74. 